\title{
Communication Framework for Tele-rehabilitation Systems with QoS Guarantee
}

\author{
Nikolay Dandanov ${ }^{1}$, Plamen Semov ${ }^{1}$, Antoni Ivanov $^{1}$, Nicole Christoff ${ }^{1,2}$, Vladimir Poulkov ${ }^{1}$ \\ ${ }^{1}$ Technical University of Sofia, Faculty of Telecommunications, Sofia 1756, Bulgaria \\ ${ }^{2}$ Aix-Marseille Université, CNRS, LSIS UMR 7296, France
}

\begin{abstract}
Nowadays, there is great interest in developing telemedicine technologies. These technologies will provide health care from distance swiftly and lightly, as well as reduce the expenses for activities, such as rehabilitation. Telemedicine applications are possible due to the advances in information technologies and the ever-expanding communication network infrastructure. The network characteristics bandwidth, packet loss, latency, jitter and policies are crucial for the performance of tele-rehabilitation systems. Because in tele-rehabilitation human lives are at stake, guaranteeing the Quality of Service (QoS) is important. In this paper, an approach for assuring the QoS for information transmission in tele-rehabilitation systems is proposed.
\end{abstract}

\section{Introduction}

In the recent years, a great advancement in the development of technological and rehabilitation methods was observed. By combining rehabilitation and the advancements in Information and Communications Technologies (ICT), tele-rehabilitation arises. This is a subpart of telemedicine, consisting of a system for controlled rehabilitation "via the Internet" - an actual application possibility with a promising development in the future. A tele-rehabilitation system is a bilateral system, consisting of master and slave devices, which interact through a bidirectional communication channel, as shown in Figure 1.

Various communication media can be used in telerehabilitation: transmission lines, wireless transmission or the Internet. Depending on the different environments, based on their performance, a decision for using TCP (Transmission Control Protocol) or UDP (User Datagram Protocol) for transporting the information has to be made.

TCP provides reliable two-way communication and guarantees data delivery at the cost of retransmissions and long timeouts that are detrimental to the performance of real-time applications, such as teleoperation. UDP does not require reception acknowledgments, eliminating unnecessary waiting time, which makes it appealing for teleoperation.

In tele-rehabilitation, improved quality video-assisted telecommunication services and devices, such as videoconferencing, webcams and telepresence, assist in treatments. Sensors and body monitoring data, voice, sounds, medical images and information related to telehaptics (the sense of touch), as well as virtual reality, can also be transmitted for rehabilitation purpose. Moreover, with the massive utilization of portable smart terminals, such as smartphones, laptops and tablets, web-based approach and mobile applications are becoming ever more common and can potentially be used for telerehabilitation.

In tele-rehabilitation, human lives are at stake. Hence, the consequences of an error or delays in information transmission could be serious. This is the reason why reliable techniques of networking must be developed and applied [1]. Particular stress should be laid on the importance of availability and reliability of the communication networks. Interruptions in network operation, e.g. introduced by a power cut, could cause errors or delays in the transmitted data. These specifics pose strong requirements for the bandwidth, delay, jitter, reliability and availability of the network connection for tele-rehabilitation.

Five fundamental network characteristics are crucial for the performance of tele-rehabilitation systems.

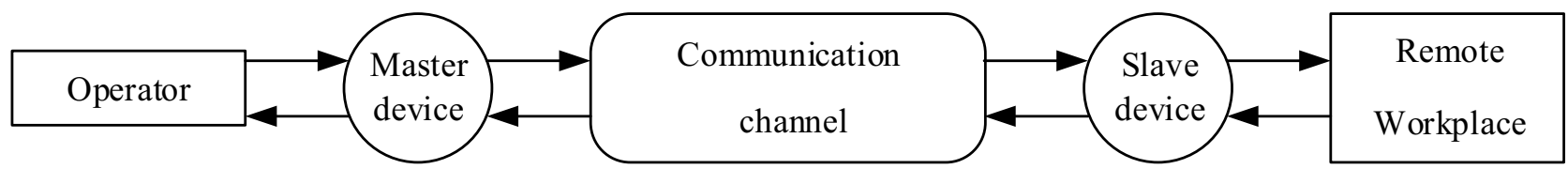

Fig. 1. Tele-rehabilitation concept

\footnotetext{
* Corresponding author: $\mathrm{n}$ dandanov@tu-sofia.bg
} 
Bandwidth is a fundamental requirement that there is enough capacity in a network path for all of the packets to get through unimpeded. Packet loss is the amount of packets, which do not arrive correctly at their destination, and it should be less than 1-2\%. Latency is the time delay between an event occurring on one site and the remote end seeing it. For a voice transmission with good quality, a latency of less than $150 \mathrm{~ms}$ is needed. Jitter is the time variation of latency among the packets, which can result in packets arriving in different order than transmitted. Jitter should be under $50 \mathrm{~ms}$. Policies are introduced by network elements like firewalls and Network Address Translation (NAT) devices. If the traffic is encrypted for security, some additional aspects should also be considered.

The rest of the paper is organized as follows. In the next section, some telecommunication approaches for tele-rehabilitation purposes ensuring the required QoS are considered. We present the problem definition and motivations in section 3 . In section 4 , various categories of telemedicine data and their Quality of Service (QoS) requirements are described. The proposed approach for assuring the QoS for information transmission in telerehabilitation systems is described in section 5. Finally, we conclude the paper in section 6 .

\section{State of the art}

In this section, some telecommunication approaches for tele-rehabilitation purposes guaranteeing the required QoS are considered.

In [2] to overcome instability, caused by unknown and varying time delay, an event-based teleoperation system is employed for synchronization of the robot and the operator. The developed teleoperation system could be easily adjusted to be useful for a tele-rehabilitation robot. UDP is used for sending operator commands and forcing feedback. Additionally, the size of packets for UDP compared to TCP puts less load on the communication channel. To avoid breakings in the control cycle, a server application monitors the control cycle's duration.

The effect of time delay can be reduced with latency tolerant control methods as presented in [3]. The proposed architecture is evaluated with three models of the system components: a master device, a slave device and a communication system. The master includes a controller and the human operator, which are connected via a high-delay medium to the slave model. The master side is where the "human operator" or the replacing automatic control device is located. The surgical staff can be found here, providing the control signals for the actuators of the slave. The communication system includes a transmitter, a receiver and a communication medium. In the deriving cascade setup, the time delay can be partially alleviated using appropriate predictive controllers, tuned to the master and slave systems [3].

In [4], the authors discuss the importance of provisioning QoS and information security in the field of medical care, focusing on the use of data communications, Internet, cloud and Smart Ubiquitous
Computational Devices (SUCDs). They present possible scenarios, related to remote control of Medical Surgical Robots via the Internet. The main idea is to lay stress on the importance of QoS provisioning and cyber security in the field of telemedicine, which also applies to telerehabilitation.

To assess the convenience of the remote telelaboratory technique, different configuration architectures have been tested in [5]. For each of these configurations, experiments have been performed, consisting of 400 remote movements of a robot. These experiments use TCP and UDP protocols. Performance details, obtained from these experiments, show that the configuration with UDP is fastest and the most convenient one for traditional tele-robotic applications. A new software protocol, called Interoperable Telesurgical Protocol (ITP), has been developed, which is open and meant for sharing. The first data interface in ITP is a packet of 84 bytes, using the UDP network protocol for fast transmission. The small size of the ITP packet makes it suitable for high packet rates.

In [6], the Secure ITP enciphers communications between the master and slave. The communications use TLS (Transport Layer Security), DTLS (Datagram TLS) protocols and the AES (Advanced Encryption Standard) cipher.

Analysis of the possibilities for providing real-time services for bilateral teleoperation systems on existing wireless infrastructures, using only application layer measurements without any information from the network layer, is presented in [7]. Since teleoperation systems should have a video feedback channel, the influence of video transmission on the transmission quality in the other two periodical channels is analysed. Based on the delay variation measurements of the two opposite directions, data channels identify WLAN (Wireless Local Area Network) problems.

The packet latency is observed and analysed in [8]. Teleoperation latency becomes noticeable to human users for delays above about $200 \mathrm{~ms}$, starts to be annoying at around $500 \mathrm{~ms}$, and significantly impacts the performance of tasks at $1 \mathrm{~s}$ and longer. In order to provide true tele-surgical care, software systems need to be in place to protect the integrity of the signal and minimize the latency of data transmission between the surgeon and the patient. One way to protect remote surgery's connectivity is to send information over multiple communication pathways or routes.

An investigation into the use of SIP (Session Initiation Protocol) protocol for a telemedicine transition system was proposed in [9]. According to standard computing platforms and operating systems, it is easier to integrate SIP protocol into the application program. Second, SIP is an extensible protocol. Therefore, it is able to run a wide range of applications with a variety of media formats and loads. In the SIP protocol, a number of criteria have been developed for instant messaging, resource management, media streaming (video) and online friend management applications.

In [10], a robotic tele-rehabilitation system has been developed to perform both interactive and cooperative bilateral rehabilitation. The system has been evaluated 
using four computer games, each with different specification of the data transmission and event management. In addition, robotic teleoperation techniques have been implemented to evaluate the performance and stability of the force reflecting strategy in the cooperative bilateral configuration. The internet communication between the robots has been performed through sockets, using the TCP/IP protocol stack.

A classification of the security threats against mobile surgical tele-robotic systems is presented in [11]. Communication networks security is an active research area and many existing strategies may be also applicable to tele-rehabilitation systems. Identifying those, as well as developing novel, specialized mitigation strategies, and analysing their impact on system's performance, security and safety, is an emerging research question.

\section{Problem Definition}

Based on the specifics of tele-rehabilitation and the resulting requirements on the network performance and QoS, a method for conforming to these demands for information transmission in tele-rehabilitation systems should be provided. The approach has to satisfy the requisites for bandwidth, latency, packet loss, jitter, policies, reliability and availability of network connection.

\section{QoS Requirements}

The data for telemedicine, which is transferred through a communication channel, can be divided into five main categories with different QoS demands:

- Voice;

- Video;

- Data from sensors;

- Teleoperation;

- Virtual reality.

\subsection{Voice over IP (Internet Protocol)}

In order to digitize human voice effectively, a sampling rate of $8 \mathrm{kHz}$ with 8 bits per sample is needed, which results in a minimum data transfer rate of $64 \mathrm{kbps}$ (kilobits per second). The delay must be less than $100-$ $150 \mathrm{~ms}$. Some notable low bit rate compression algorithms used are ITU G.723.1 and G.729A. It is common to use silence compression to reduce the payload dramatically.

\subsection{Video}

The type of video, which is most important in telemedicine, is the video, which is transmitted in realtime. The throughput in raw format, without compression, varies depending on three main features resolution, frame rate and bits per pixel. For video with resolution $1920 \times 1080$ pixels, 30 frames per second, RGB (Red, Green and Blue) color components and 8 bits per pixel per color, a total video stream with data rate of $1.5 \mathrm{Gbps}$ in raw format is produced. Techniques, which compress individual frames, have been developed. In addition, motion prediction is used to compress the data even further. Most of the time, the throughput for video transmission is dependent mainly on the motion within the images. Factors, such as resolution and scanning rates, are not so relevant. A full-resolution HDTV (HighDefinition TeleVision) stream, compressed with the MPEG2 method, requires $19.3 \mathrm{Mbps}$ of data bandwidth. For online video streaming with $720 \mathrm{p}$ resolution, a 2.4 Mbps channel is required.

Every computation for compressing and decompressing the video adds delay to the total transmission delay. The greater the compression ratio used, the greater the latency introduced. The total delay requirement for video transmission is less than $150 \mathrm{~ms}$.

\subsection{Data from sensors}

For this type of information, the requirements for throughput and delays are not so stringent. However, high requirements for data integrity are set. Efficient transport protocols are needed to provide correctly received sensors' data.

\subsection{Teleoperation and Virtual reality}

This category includes a mix of video, voice, text, sensors data and remote device commands, which are to be multiplexed and transferred through the communication channel simultaneously in real-time. A list of provisional network requirements for teleoperation and virtual reality can be identified. These QoS requirements include [1], [4]:

- reliability and integrity;

- an acceptable end-to-end delay;

- multiplexing of various data rates resulting in high network bandwidth;

- low data error rate (BER - Bit Error Rate);

- low latency;

- constant jitter.

Studies show that the acceptable limit of time delay in terms of a surgeon's perception of safety is roughly $330 \mathrm{~ms}$ [1]. A study in [1] shows how the interactive Internet gaming is affected by the end-to-end delay. The results are presented in Table 1 .

Table 1. Perception of interactive Internet gaming as a function of end-to-end delay [1]

\begin{tabular}{|c|c|c|c|c|c|}
\hline $\begin{array}{c}\text { End- } \\
\text { to-end } \\
\text { delay }\end{array}$ & $\begin{array}{c}<\mathbf{5 0} \\
\mathbf{m s}\end{array}$ & $\begin{array}{c}\mathbf{5 0 - 1 0 0} \\
\mathbf{m s}\end{array}$ & $\begin{array}{c}\mathbf{1 0 0 - 1 5 0} \\
\mathbf{m s}\end{array}$ & $\begin{array}{c}\mathbf{1 0 0 -} \\
\mathbf{1 5 0} \mathbf{m s}\end{array}$ & $\begin{array}{c}>\mathbf{2 0 0} \\
\mathbf{m s}\end{array}$ \\
\hline $\begin{array}{c}\text { Per- } \\
\text { cep- } \\
\text { tion }\end{array}$ & $\begin{array}{c}\text { Excel- } \\
\text { lent } \\
\text { game- } \\
\text { play }\end{array}$ & $\begin{array}{c}\text { Good } \\
\text { game- } \\
\text { play }\end{array}$ & $\begin{array}{c}\text { Noticea- } \\
\text { bly } \\
\text { decreased } \\
\text { gameplay }\end{array}$ & $\begin{array}{c}\text { Signifi- } \\
\text { cantly } \\
\text { effected } \\
\text { gamep- } \\
\text { lay }\end{array}$ & $\begin{array}{c}\text { Intole- } \\
\text { rable } \\
\text { game- } \\
\text { play }\end{array}$ \\
\hline
\end{tabular}




\section{Approach for assuring QoS for information transmission in tele- rehabilitation systems}

Because of the various transport protocols and telemedicine technologies existing, we propose adaptable and scalable framework for tele-rehabilitation. Its main functions are:

1) providing abstraction and generalization of the transmitted data among telemedicine devices from different vendors. This way, the network infrastructure is transparent;

2) Data Tunneling for meeting various QoS requirements;

3) M2M communication capabilities for telerehabilitation devices from different vendors;

4) General and scalable application software for all telemedicine technologies;

5) Adaptation of the transport layer for reaching target QoS requirements.

The proposed framework is illustrated on Figure 2.

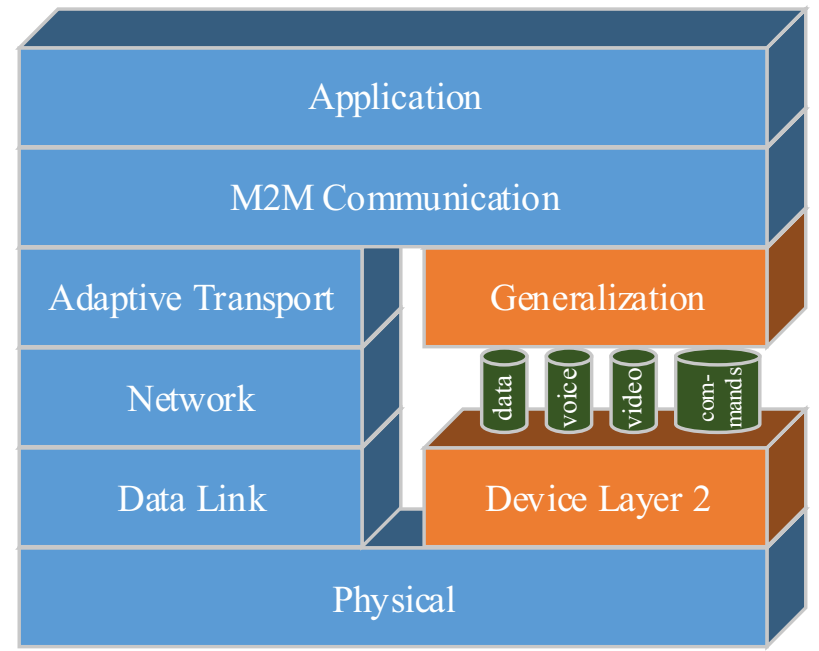

Fig. 2. Proposed framework for assuring QoS for information transmission in tele-rehabilitation systems

The layer "Generalization" has the main responsibility to set the parameters for each data stream generated by a tele-rehabilitation device. It controls telerehabilitation devices, or more specifically - their output data streams. Another main responsibility is to adapt or translate different teleoperation and sensors data streams, which are sometimes unique for the device itself. The layer gives a compatibility function for all telerehabilitation devices to work together.

The main responsibility for the "M2M Communication" layer is to give tele-rehabilitation devices abilities to share and request data from each other, which is needed for taking an overall decision by a given tele-rehabilitation device. This functionality can be realized as a sublayer of the Application layer.

"Application" layer is the man-machine interface, which sets QoS requirements for the data streams. It works also like a SIP server to negotiate data stream parameters between different vendor-specific or technology-specific devices. Another function is to track communication KPIs (Key Performance Indicators), such as delays, throughput, error rate, latency, etc. This acts as a feedback for the "Generalization" and "Adaptive Transport" layers, so they can further modify data and communication parameters and thus meet QoS requirements.

The "Adaptive Transport" layer has the task to fulfill the QoS requirements for every data stream on the transport layer by using various transport protocols existing or novel. For transporting information, which is insensitive to delays but should be received with no errors, TCP can be used, for example for controlling a remote workplace or receiving measurements from sensors.

Because of the broad development of the Internet and associated IP networks and protocols, this is one of the candidates for providing such transport. In this case, one appropriate protocol for transporting media in real-time is SIP in addition with Session Description Protocol (SDP) and Real-time Transport Protocol (RTP) or Secure RTP (SRTP). For a transport protocol, either TCP, UDP, Stream Control Transmission Protocol (SCTP) or any other suitable implementation can be leveraged.

By implementing this multilayer framework, we separate the communication channel functions from the tele-rehabilitation devices functions. Hence, telerehabilitation technologies can be developed independently from the network infrastructure. This method will be a common interface for all telerehabilitation technologies, providing network communication capabilities.

With the use of data tunneling, QoS requirements for the transmission of different information types are met. For meeting stringent QoS requirements, a tailored protocol on the transport layer can be developed. The M2M communication capabilities assure compatibility between tele-rehabilitation devices from different vendors. This unity enables general and scalable application software for all telemedicine technologies.

The information with particular requirements for network performance can be separated into different traffic flows. Each of these flows should use a distinct stack of protocols, tailored to fulfilling the requirements posed by this type of information.

As the technologies and services in the networks evolve, backward compatibility should be maintained, so that devices from both newer and older generations can interoperate together.

\section{Conclusions and Future Work}

The proposed communication framework for telerehabilitation systems with QoS guarantee provides abstraction and generalization of the transmitted data among various tele-rehabilitation devices. Hence, the network infrastructure is transparent to the communicating devices. Data tunneling is leveraged for meeting various QoS requirements. M2M communication capabilities are added for 
interoperability. Adaptation in the transport layer protocols is a means to reach target QoS requirements.

As a result, the framework can serve to develop generalized and scalable application software for all telerehabilitation technologies.

The communication between tele-rehabilitation systems has to be designed in such way, so that it allows compatibility between devices from different vendors and of various generations, and provides openness of the underlying protocols and methods.

The next steps in the development of the common communication framework for tele-rehabilitation systems would include a more elaborate selection and design of the protocols in each layer should be performed. After a protocol stack has been chosen, the integrity of the whole stack shall be tested and evaluated. This can be done by simulating the interoperation of separate protocols and devices or by implementing the stack in tele-rehabilitation systems and devices.

\section{Acknowledgment}

This work is supported by Project № BG05M2OP001-2.009-0033-C01.

\section{References}

1. J. Marescaux, J. Leroy, M. Gagner, F. Rubin, D. Mutter, M. Vix, S. Butner, M. K. Smith. Transatlantic Robot-Assisted Telesurgery. Nature, 413, 379-380 (2001)

2. I. Petrović, F. Kolonić, P. Korondi. Internet Based Teleoperation for Cooperative Navigation and Manipulation. Mec. Eng., 6, 356-368 (2009)

3. S. Jordán, A. Takács, I. Rudas, T. Haidegger. Modelling and Control Framework for Robotic Telesurgery (2013)

4. M. Smithwick, Network Options for WideArea Telesurgery. Telemed. and Tel, 1 (3), 131-38 (1995)

5. R. Marín, P. J. Sanz, P. Nebot, R. A. Wirz. Multimodal Interface to Control a Robot Arm via Web: A Case Study on Remote Programming. IEEE Transactions on Industrial Engineering, 52 (6), 1506-1520 (2005)

6. G. Lee, B. Thuraisingham. Cyberphysical systems security applied to telesurgical robotics. Comp. St. \& Int. 34, 225-229 (2012)

7. P. Haller, T. Vajda, Z. Szanto, L. Marton. Communication Performance Evaluation of Bilateral Teleoperation systems Implemented over WLAN. http://www.ms.sapientia.ro/ martonl/Researc h_TE_Documents/WLAN_Meas.pdf (2013)

8. T. Lendvay, J. Rosen, B. Hannaford. Telerobotics: Its Future in Clinical Application. Pediatric Robotic and
Reconstructive Urology: A Comprehensive Guide, Blackwell Publishing Ltd., 314-327 (2012)

9. T. Long. Telemedicine Transmission System Design Research Based on Session Initiation Protocol. Soft. Eng., 9, 298-307 (2015)

10. A. A. Siqueira, K. P. Michmizos, H. I. Krebs. Development of a robotic system for bilateral telerehabilitation. In Proceedings of 22nd International Congress of Mechanical Engineering (COBEM 2013), Ribeirão Preto, SP, Brazil. ABCM, Rio de Janeiro, Brazil, 8427-8436 (2013)

11. T. Bonaci, H. J. Chizeck. Surgical Telerobotics Meets Information Security. RSS 2012 Workshop on Algorithmic Frontiers in Medical Robotics: Manipulation in Uncertain, Deformable, Heterogeneous Environments. Sydney, Australia (2012) 